Musées, Patrimoine et Culture scientifiques et techniques

$138 \mid 2011$

novembre - décembre 2011

\title{
Le diagnostic des infestations en milieu patrimonial : approches techniques et méthodologiques
}

Infestation diagnosis in the heritage field: technical and methodological approaches

Fabien Fohrer

\section{OpenEdition}

Journals

Édition électronique

URL : https://journals.openedition.org/ocim/988

DOI : 10.4000/ocim.988

ISSN : 2108-646X

Éditeur

OCIM

Édition imprimée

Date de publication : 1 novembre 2011

Pagination : $31-40$

ISSN : 0994-1908

Référence électronique

Fabien Fohrer, « Le diagnostic des infestations en milieu patrimonial : approches techniques et méthodologiques », La Lettre de I'OCIM [En ligne], 138 | 2011, mis en ligne le 01 novembre 2013, consulté le 21 septembre 2021. URL : http://journals.openedition.org/ocim/988 ; DOI : https://doi.org/ 10.4000/ocim. 988 


\section{Le diagnostic des infestations en milieu patrimonial : approches techniques et méthodologiques}

Fabien Fohrer *

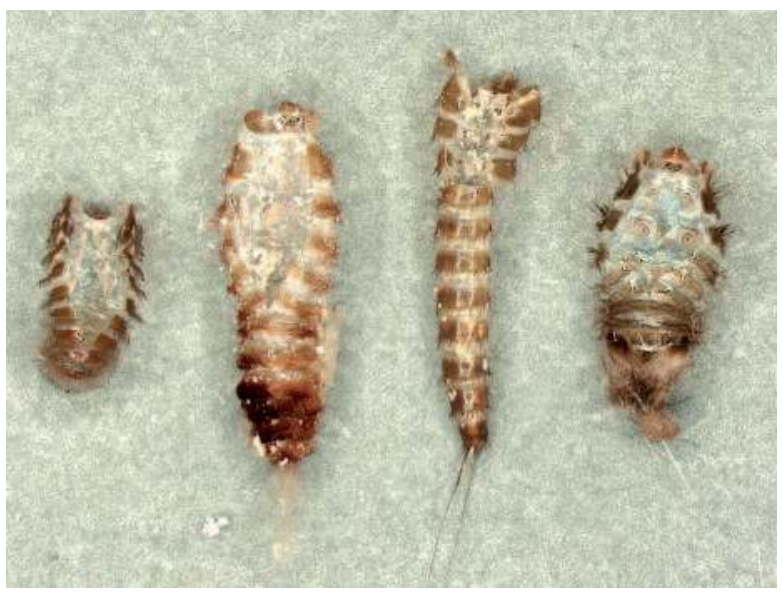

Exemples de dépouilles larvaires de la famille des Dermestidae, de gauche à droite : Thylodrias contractus, Trogoderma versicolor, Attagenus unicolor, Anthrenus verbasci. ๑ CICRP

* Fabien Fohrer est entomologiste et microbiologiste au service de Conservation préventive du Centre interrégional de Conservation et Restauration du Patrimoine de Marseille fabien.fohrer@cicrp.fr
La mise en place dans les institutions muséales de moyens spécifiques de lutte contre les insectes passe - comme le montre cette contribution - par une analyse précise des facteurs favorisant la présence de ces insectes ainsi que par une connaissance des matériels et des différentes techniques de repérage et d'identification.

\section{Introduction}

Les insectes peuvent causer des dégradations irrémédiables tant sur les œuvres présentes dans les lieux muséographiques que sur les structures et bâtiments qui les abritent. Ces infestations ont pour origine différentes espèces d'insectes qu'il faut détecter et identifier afin de mettre en œuvre les actions curatives et préventives adaptées.

Pour ce faire, la première action à entreprendre est la recherche des espèces d'insectes incriminées dans les dégradations des œuvres. Cette recherche réalisée au sein des œuvres et de leur environnement est un préambule à toute action préventive et curative.

La connaissance de l'espèce incriminée dans les dégradations permet grâce à son régime alimentaire (xylophage, nécrophage..), de remonter jusqu'au type de matériau qu'elle infeste (bois, papier, cuir, plume...) et ainsi d'effectuer une recherche plus active sur le type d'œuvre pouvant être infestée pour permettre de remonter jusqu'à la ou les sources d'infestation.

Pourquoi et comment les insectes sont-ils présents dans les lieux muséographiques?

Le but du diagnostic est de comprendre et de rechercher les causes de la présence des insectes néfastes dans les 


\section{Une base de données consacrée aux insectes du pairimoine culfurel}

Le CICRP a dernièrement mis en ligne une base de données consacrée aux insectes dangereux pour le patrimoine culturel (wwwl.montpellier.inra.fr/CBGP/insectes-dupatrimoine). Elle est le fruit d'une collaboration entre le CICRP et l'Institut national de la Recherche agronomique de Montpellier et permet de faciliter la reconnaissance des insectes rencontrés dans les lieux patrimoniaux.

\section{Que contient cette base de données ?}

Pour chacune des espèces d'insectes recensées, plusieurs entrées sont proposées telles que les noms communs et les noms scientifiques, les matériaux infestés, les séquences moléculaires, les clés d'identifications (dichotomiques et visuelles)... Ces entrées renvoient toutes à une fiche descriptive pour chacune des espèces. En plus de textes généraux sur la nomenclature, la classification et la présentation des ordres et des familles, cette base de données renseigne également sur la reconnaissance, la biologie, la fréquence et la répartition de chacune des espèces mentionnées.

Enfin un glossaire recense le vocabulaire entomologique et technique utilisé dans la base, afin de permettre une meilleure compréhension des textes et descriptions, quel que soit le niveau des connaissances techniques ou scientifiques du consultant.

\section{Le futur de l'identification des insectes : l'identification des insectes par l'approche moléculaire}

Une des spécificités de cette base de données est la possibilité de pouvoir identifier un insecte à partir de son ADN. Par rapport à l'identification morphologique, l'utilisation des caractères moléculaires pour l'identification taxonomique présente de nombreux avantages. En effet, cette technique permet la réalisation d'identification fiable de tous les stades de développement des insectes (œufs, larves, nymphes...) et la détection des espèces cryptiques (peu différenciées). Elle ne nécessite pas d'être formé à la maîtrise des terminologies morphologiques variant selon les taxas et les stades de développement.

Pour atteindre ce but nous établissons actuellement une banque de données à partir de séquences d'ADN extraites de spécimens exactement identifiés par des entomologistes. Dans quelques années, nous pensons que cette technique permettra l'identification des insectes de manière routinière. Les techniques de séquençage de nouvelle génération permettront la production de millions de séquences instantanément, ce qui permettra de réduire les coûts d'identification locaux et sur les œuvres. Ces causes peuvent avoir malheureusement de multiples et interdépendantes origines. Voici les raisons les plus courantes de leur présence et de leur prolifération dans les lieux muséographiques.

Les facteurs favorisant la pénétration des insectes dans un bâtiment

\section{L'étanchéité des bâtiments}

Une des causes majeures de la présence des insectes dans les lieux muséographiques est la perméabilité des bâtiments à leurs entrées. En effet, bien souvent les insectes pénètrent à l'intérieur des bâtiments du fait d'une mauvaise étanchéité des portes et des fenêtres ou de toute autre ouverture importante. Ces insectes peuvent se trouver présents à l'extérieur d'un bâtiment au sein des espaces verts (fleurs), dans la matière organique, ou dans les nids d'oiseaux par exemple. Des solutions simples peuvent être apportées comme la pose de moustiquaires aux fenêtres et de balais anti-intrusion au bas des portes ou encore la réfection des maçonneries qui auraient permis leur entrée.

\section{Le don et le prêt des œuvres}

Le don et le prêt des œuvres sont des sources extérieures d'infestation non négligeables qu'il est important de prendre en compte par leurs mises en quarantaine (avant qu'elles intègrent ou réintègrent les lieux de stockages). Une inspection doit être réalisée sur les œuvres et au niveau de leur conditionnement afin de repérer d'éventuelles traces ou la présence d'insectes (morts et vivants).

\section{Les facteurs favorisant la prolifération des insectes}

Parmi les nombreux facteurs qui favorisent la prolifération des insectes au sein d'une institution, voici indiqué, ci-dessous, les plus importants.

\section{L'entretien des locaux et des collections}

La poussière, par sa composition (débris végétaux et animaux de toutes sortes, présence de pollens, de spores de moisissures et suies industrielles acides...), est un support nutritif très important qui favorise la prolifération des insectes. Souvent très acide et absorbante, l'humidité est un état 


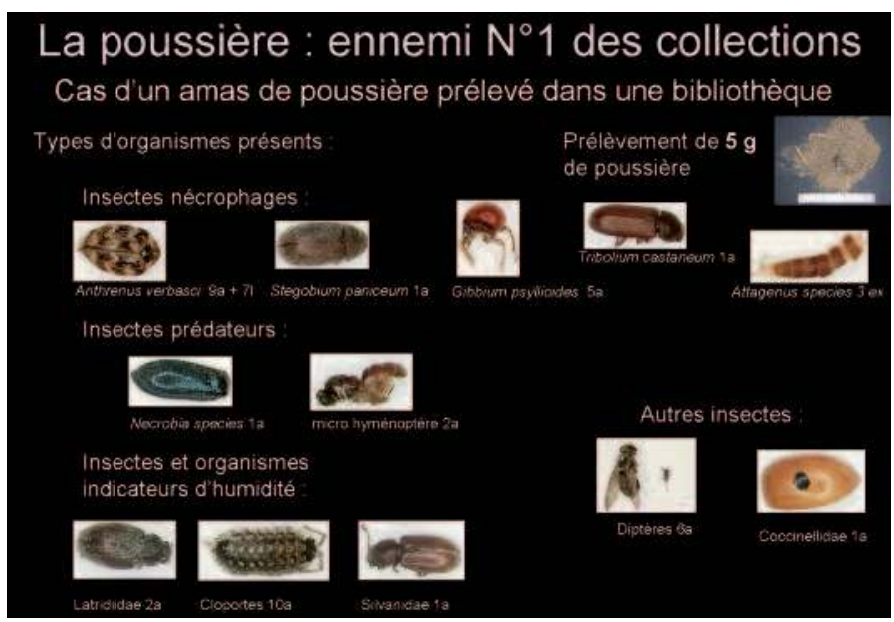

Espèces d'insectes rencontrées dans un prélèvement de $5 \mathrm{~g}$ de poussière au sein $\mathrm{d}^{\prime}$ une bibliothèque.

(c) CICRP

très favorable à la germination des spores de moisissures dont certaines espèces se nourrissent en priorité (psoques, poissons d'argent, blattes...) tout en permettant l'implantation d'insectes nécrophages et psychophages (qui se nourrit de miettes). Lorsque cet empoussièrement est lié à des conditions climatiques défavorables (principalement l'augmentation du taux d'humidité relative), il favorise grandement la vitesse de développement et la prolifération des insectes.

\section{Le rangement au sein des espaces}

Une des causes majeures de la prolifération des insectes au sein d'une institution est l'existence de lieux, endroits ou coins « laissés à l'abandon » dans lesquels sont entreposés de nombreux matériaux en attente d'être utilisés. Ces endroits de stockage provisoires deviennent vite des lieux de «fourre tout », oubliés de tous et qui permettent bien souvent la prolifération des insectes à l'abri de tous regards. Bien souvent les conditions qui règnent dans ces endroits (caves, greniers et débarras) sont déplorables (humidité et empoussièrement excessifs).

\section{Une lutte mal maîtrisée}

Parfois certaines actions entreprises au sein d'une institution peuvent avoir comme conséquence la prolifération des insectes de manière tout à fait involontaire. Tel est le cas par exemple de la lutte contre les rongeurs (pose d'appâts qui permet de les éradiquer), qui mal gérée (appât oublié dans un coin et non relevé) peut permettre la prolifération d'insectes néfastes qui se dispersant dans l'environnement se retrouvent par la suite sur les œuvres.

Parmi les insectes présents dans ces appâts, on retrouve des insectes nécrophages de la famille des Dermestidae (Anthrenus verbasci, Anthrenocerus australis, Reesa vespulae...), des insectes polyphages de la famille de Anobiidae (Stegobium paniceum et Lasioderma serricorne) ainsi que des psoques (Liposcelis species).

\section{Les impacts d'un nettoyage non adapté}

Un entretien des sols à grande eau peut avoir également comme conséquence l'implantation et la prolifération de certains insectes : l'eau peut par exemple imbiber les plinthes en bois et générer des conditions favorables à leur développement (cas des lépismes et de certaines espèces d'insectes à larve xylophage comme le Curculionidae Hexathrum exiguum).

\section{L'apport extérieur de nourriture}

Enfin l'apport de nourriture au sein d'une institution par le biais du personnel (coins repas, penderie) ou des visiteurs (morceau de nourriture laissé dans les salles d'exposition et pièce où sont entreposés les objets trouvés par exemple) peuvent contribuer à l'implantation, à la prolifération et à la dissémination d'insectes dangereux pour les collections.

Dans ces situations on rencontre fréquemment la vrillette du pain Stegobium paniceum, la mite des vêtements Tineola bisselliella et la mite alimentaire Plodia interpunctella.

\section{Le repérage de l'activité et la présence des insectes}

Les insectes néfastes aux collections sont généralement de petite taille (inferieure à $8 \mathrm{~mm}$ ), très discrets (peu visibles la journée), et les dégâts observés sont réalisés la plupart du temps par les larves, les adultes ne s'alimentant pas et ont une durée de vie courte ( 1 à 2 mois).

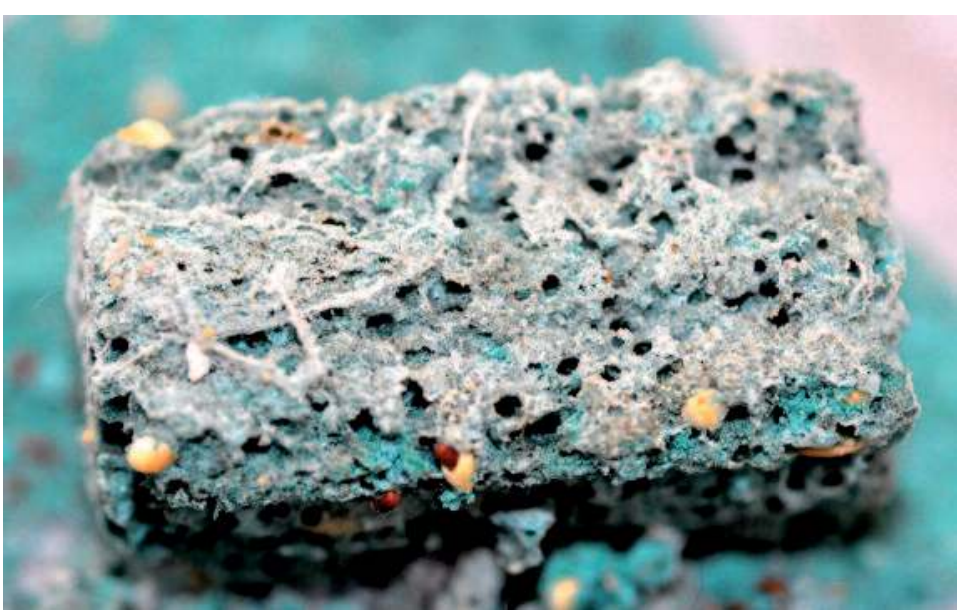

Forte infestation (Stegobium paniceum) d'un block raticide utilisé dans la lutte contre les rongeurs (trous d'envol, vermoulures et insectes adultes). () CICRP 


\section{Au niveau des œuvres}

Leur repérage s'effectue bien souvent par l'observation des traces d'activités qu'ils occasionnent sur les œuvres (trous d'envols, vermoulures, perte de matière, dépouilles larvaires) ainsi que par la présence d'adultes et de larves sur les matériaux.

Le repérage des dépouilles larvaires indique bien souvent une activité larvaire spécifique à la famille des Dermestidae (Anthrènes, Attagènes, Trogodermes) alors que les fourreaux indiquent l'activité des lépidoptères de la famille des Tineidae principalement (mite des vêtements et mite à fourreaux).

\section{Au niveau de l'environnement}

Les insectes adultes peuvent se repérer volant dans l'environnement ou rampant sur le sol, en général du printemps à l'automne, l'hiver étant la saison de latence des insectes. Lors du diagnostic, et pour détecter leur présence, il est important de les rechercher au niveau des sources lumineuses naturelles (rebords et bas de fenêtres) et des sources lumineuses artificielles (diverses lampes et néons). On pourra les repérer assez aisément dans les endroits peu fréquentés comme les caves, les greniers, les vides sanitaires, les coins sombres et sous les planchers où ils peuvent se développer en toute discrétion.

Les pièges naturels que sont les toiles d'araignées et la poussière peuvent également indiquer leur présence.

\section{Les matériels utilisés lors du diagnostic}

Les insectes étant discrets et de petite taille, il est important de se munir de matériels adaptés afin de les repérer.

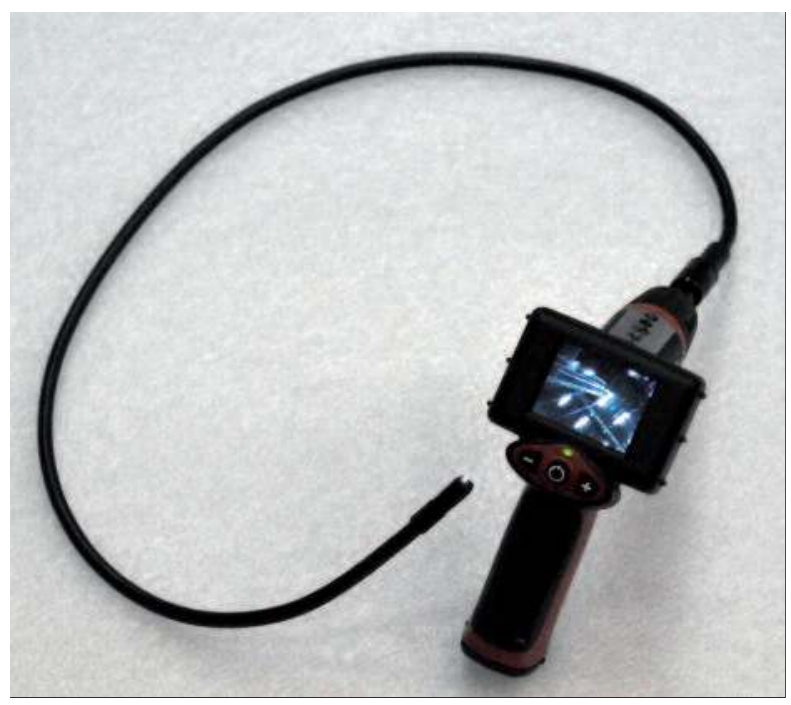

L'endoscope, appareil utilisé pour visualiser les endroits peu accessibles à la recherche de trace d'activité d'insectes.

$\odot$ CICRP

\section{Le matériel d'inspection}

Il est principalement constitué d'une lampe à éclairage assez puissant et d'une loupe afin de repérer le moindre insecte ou la moindre trace indiquant leur présence. Ce repérage est d'autant plus aisé lorsque les mains sont libres ce qui permet de soulever et déplacer certaines parties des œuvres (plis d'un tissu par exemple). Le port d'un casque frontal muni d'une loupe et d'un éclairage est l'outil le plus adapté pour réaliser ce type de diagnostic.

Afin de réaliser un diagnostic dans les endroits peu accessibles, du matériel plus adapté peut être utilisé tel que le vidéoscope (ou endoscope et fibroscope). Cet appareil portable est constitué d'une fibre optique semi-rigide d'une longueur maximale de 8-10 m, au bout de laquelle est installée une caméra et un système d'éclairage par LED qui permet la réalisation de prises de vue et de films. Il est utilisé pour rechercher la présence d'insectes ou de trace d'activité au dos de retable ou au revers des peintures dans les lieux et réserves encombrés, sous les parquets et rayonnages et dans les locaux techniques et vides sanitaires.

D'autres matériels de visualisation peuvent être utilisés afin d'affiner le diagnostic tel qu'un appareil photographique avec zoom macroscopique, un petit microscope de poche éclairant (type fissuromètre) ou une paire de jumelles pour observer par exemple l'état d'infestation du bois des charpentes ou l'état d'entretien des gouttières.

\section{Le matériel de mesures climatiques}

Le diagnostic peut être précisé en réalisant des mesures de conditions environnementales à l'aide d'un thermohygromètre d'ambiance et d'un hygromètre à matériaux. Ces mesures prises à un instant donné, indiquent un désordre climatique ou un microclimat particulier qui aurait pu favoriser le développement et la prolifération des insectes. L'implantation de capteurs thermo hygrométriques (avec courbes de relevés) directement placés dans les espaces (par l'institution) permet de faire un suivi des conditions climatiques et ainsi de repérer tout changement important qui pourrait influer sur la vitesse de développement et la prolifération des insectes.

\section{Le matériel de prélèvement des insectes}

\section{Que doit-on prélever?}

Les prélèvements à effectuer doivent permettre d'apporter toutes les informations nécessaires à la détermination des espèces d'insectes mises en cause dans la dégradation des œuvres. Voici une liste non exhaustive des différents types de prélèvements et du niveau d'identification qu'ils peuvent apporter. 


\begin{tabular}{|c|c|c|}
\hline Prélèvements & Niveaux d'identification possibles & Commentaires \\
\hline Insectes adultes & À l'espèce & $\begin{array}{l}\text { L'identification à ce niveau dépend de la } \\
\text { manière dont le prélèvement est réalisé, } \\
\text { l'insecte devant être en parfait état }\end{array}$ \\
\hline Larves & À la famille, au genre voire à l'espèce & $\begin{array}{l}\text { Les larves de l'Anthrenus flavipes sont } \\
\text { reconnaissables parmi les autres espèces } \\
\text { de ce genre. }\end{array}$ \\
\hline $\begin{array}{l}\text { Dépouilles larvaires } \\
\text { et nymphales }\end{array}$ & $\grave{A}$ la famille et au genre & $\begin{array}{l}\text { Caractéristiques des coléoptères de la } \\
\text { famille des Dermestidae et des lépidoptères } \\
\text { de la famille des Tineidae }\end{array}$ \\
\hline $\begin{array}{l}\text { Vermoulures } \\
\text { et débris d'insectes }\end{array}$ & À la famille, au genre voire à l'espèce & $\begin{array}{l}\text { La vermoulure de la grosse vrillette } \\
\text { Xestobium rufovillosum est caractéristique } \\
\text { (lentille de } 1 \mathrm{~mm} \text { de diamètre) }\end{array}$ \\
\hline Poussière & De l'ordre à l'espèce & $\begin{array}{l}\text { Dépend de l'état de conservation } \\
\text { des insectes }\end{array}$ \\
\hline
\end{tabular}

Comment prélever les insectes et quels matériels utiliser?

Pour réaliser des identifications fiables, les spécimens d'insectes récoltés doivent être en parfait état de conservation (les pattes, les antennes, les cerques et les soies, appendice très fragile). Ils sont souvent indispensables pour réaliser une identification allant jusqu'à l'espèce. Bien prélever un insecte permet par la suite de réaliser une bonne identification. Voici quelques types de matériels pour la réalisation d'un prélèvement correct :

\section{PRÉLÈVEMENT D'INSECTES VIVANTS}

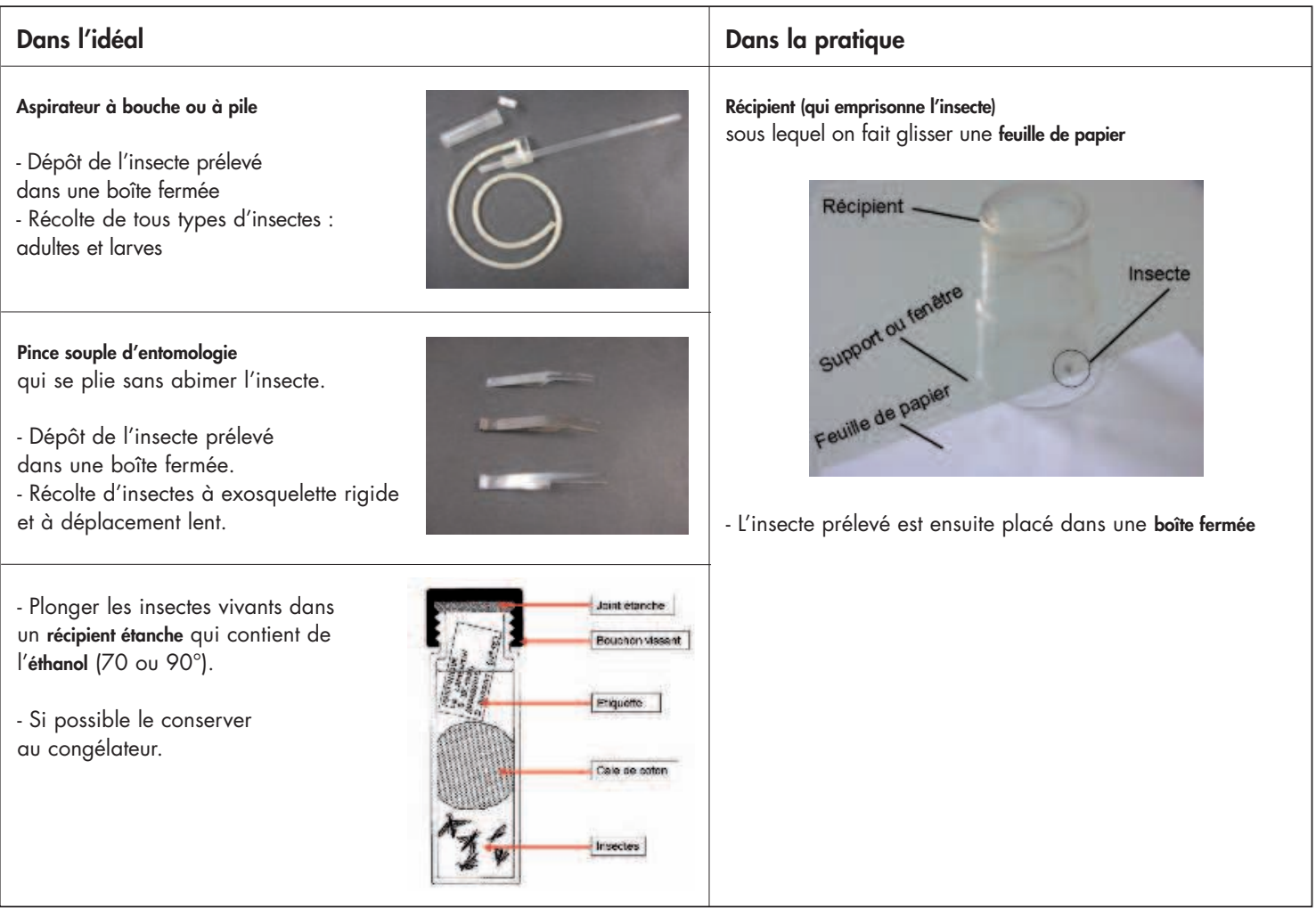


PRÉLÈVEMENT D'INSECTES MORTS

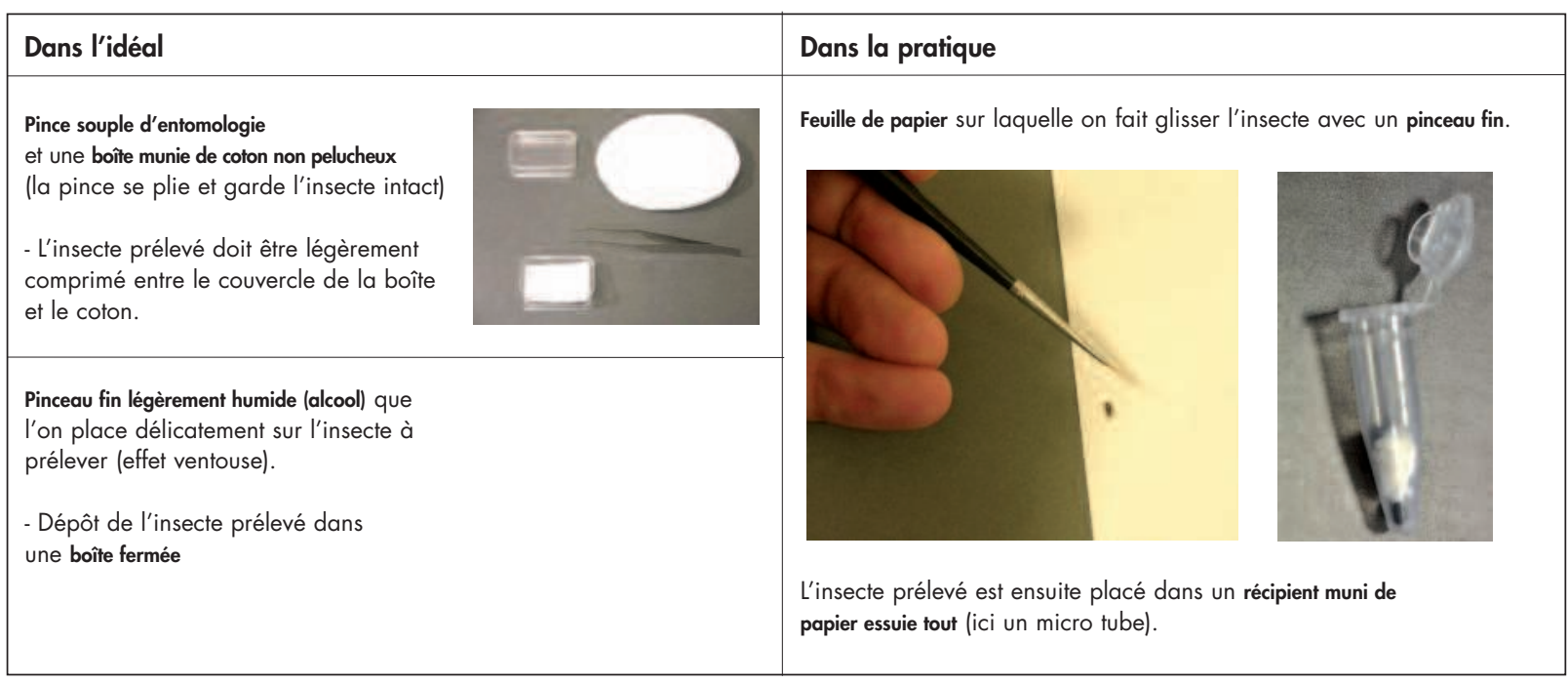

Pour le prélèvement des vermoulures, des débris et de la poussière, on procède comme pour la récolte des insectes morts (dans la pratique) ou à l'aide d'un aspirateur à pile. Tous les prélèvements réalisés doivent être renseignés avec le maximum d'informations dont : la date du prélèvement, le lieu de prélèvement (ville, musée, salle, réserve, vitrine, sol, intérieur, extérieur...), l'objet ou le matériau où l'insecte a été prélevé et le nom du récolteur.

\section{Piégeage des insectes: méthodes et matériels}

Le but du piégeage est de permettre la détection et la récupération des insectes, présents dans l'environnement de manière autonome, en vue de leur identification afin de mettre en place les actions curatives et préventives adaptées.

\section{Les pièges actifs}

Ces types de pièges présentent une attractivité particulière qui permet la capture des insectes.

\section{Les pièges lumineux}

Les insectes possèdent une forte attirance par rapport à certaines sources lumineuses (phototropisme). Le spectre d'attractivité aux rayons ultra violets (UV) diffère suivant le type d'insecte mais la grande majorité des insectes sont sensibles aux UV-A (entre $380 \mathrm{~nm}$ et $315 \mathrm{~nm}$ ). Un maximum d'efficacité est atteint aux environs de $365 \mathrm{~nm}$, notamment pour les diptères (mouches) et permet également l'attirance de quelques espèces dangereuses pour les œuvres (par exemple le Stegobium paniceum). Les insectes sont moins sensibles aux autres UV (UV-B entre $315 \mathrm{~nm}$ et $280 \mathrm{~nm}$ et UV-C entre $100 \mathrm{~nm}$ et $280 \mathrm{~nm}$ ) mais le procédé garde néanmoins son intérêt.
Ces pièges sont équipés de tube ultra violet derrière lesquels sont placés des plaques de glu, ce qui permet une détermination des insectes piégés et d'évaluer leur population à un moment donné. Ces éléments sont des indicateurs des risques potentiels pour les œuvres et permettent d'estimer l'ampleur du risque. Il existe également des systèmes électrocutant, qui ne sont pas à recommander pour le domaine patrimonial, car les insectes explosent au contact du piège ce qui exclut tout comptage et identification.

Ces pièges peuvent être posés dans les lieux de passage ou dans les réserves en fonction du type de matériaux constitutifs des œuvres. Il faut veiller à les maintenir éloignés des œuvres en raison des risques photochimiques sur celles-ci : dégradation et décoloration sur certains matériaux des objets (plumes colorées, poils, pigments...).

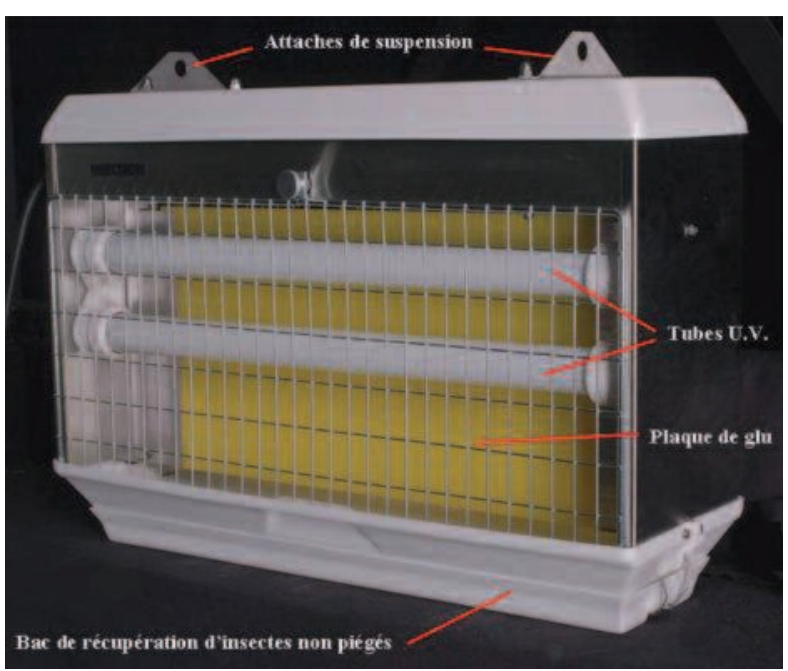

Piège à UV pouvant être suspendu au centre d'une pièce, utilisé pour le piégeage des insectes volants.

(c) CICRP 
Il existe plusieurs types de pièges : mural, suspendu, en applique. La puissance des appareils est fonction du volume à traiter. Dernièrement, sont commercialisés de nouveaux types de tubes à lumière verte $(500 \mathrm{~nm})$ qui ne produisent pas de rayonnement UV ainsi que des appareils à LED UV moins gourmands en électricité. Enfin, afin de diriger le rayonnement UV, des réflecteurs sont placés sur certains types d'appareils, ce qui permet d'obtenir une lumière indirecte. Tous ces pièges permettent uniquement la capture des insectes adultes volants.

Ces appareils demandent peu de maintenance. Il faut veiller à changer les tubes UV, une fois par an et les plaques de glu, tous les mois entre mars et septembre (période propice aux développements des infestations), tous les trois mois pour les autres mois de l'année. Il n'est pas nécessaire de changer obligatoirement les plaques de glu, si peu d'insectes s'y trouvent piégés, il suffit de les retirer de la glu à l'aide d'un solvant (type détacheur universel) pour permettre leur identification.

\section{Les pièges à phéromones sexuelles}

Les phéromones sexuelles sont des substances chimiques volatiles qui permettent d'attirer les insectes entre eux dans le but de se reproduire. Elles sont produites par un des deux sexes (généralement la femelle attire le mâle) et sont spécifiques pour une espèce donnée. Ces phéromones fabriquées par synthèse, sont constituées de plusieurs molécules complexes dont les rôles ne sont pas toujours bien connus. C'est la raison pour laquelle, pour une espèce d'insecte donnée, les différentes marques de phéromones fabriquées ne possèdent pas toutes le même pouvoir d'attractivité et qu'il est important de pouvoir les tester en amont.

Ces pièges sont constitués d'une plaque de glu au milieu de laquelle est placée une capsule de phéromone. Cette capsule doit être régulièrement changée car sa durée d'action est limitée dans le temps (environ 2 à 3 mois). Ces pièges permettent de couvrir une surface de 3 à 10 mètres linéaires. Ils peuvent être placés, soit suspendus dans l'environnement (exemple piège à mites) dans des réserves par exemple, soit placés plus discrètement sous un meuble, sous

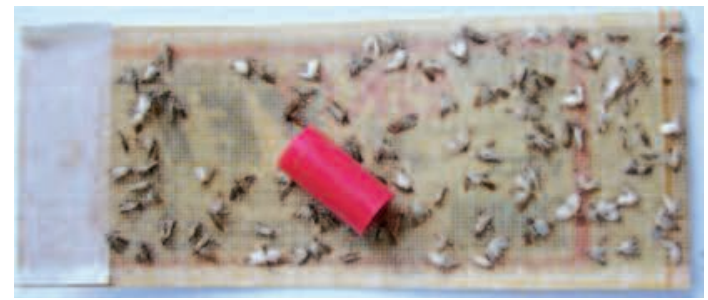

Piège à phéromone, constitué d'une plaque de glu et $d^{\prime}$ 'une capsule de phéromone (en rouge) utilisé ici pour le piégeage des mites alimentaires Plodia interpunctella. une étagère ou cachés dans une vitrine pour les salles d'exposition. Une implantation raisonnée des pièges permet de remonter jusqu'à la source d'infestation afin de prendre les mesures qui s'imposent (quarantaine et traitement). Tous ces pièges permettent uniquement la capture des insectes adultes volants.

Puisque les phéromones ont une action spécifique pour une espèce donnée, et vu le coût important et la fréquence de remplacement de celles-ci, il est primordial de bien identifier l'espèce que l'on veut piéger. Actuellement, peu de phéromones sont fabriquées pour détecter les insectes dangereux pour le patrimoine (la phéromone de la petite vrillette Anobium punctatum n'est plus commercialisée). On peut néanmoins trouver quelques phéromones pour piéger les espèces de coléoptères de la famille des Anobiidae (Lasioderma serricorne et Stegobium paniceum) et de la famille des Dermestidae (Espèces des genres Anthrenus et Attagenus). Enfin, les pièges à phéromones contre les mites (mites alimentaires de la famille des Pyralidae, des genres Plodia et Ephestia et mites à vêtement et à fourreau de la famille des Tineidae des genres Tinea et Tineola) sont particulièrement efficaces mais là encore les différentes marques de phéromones produites n'ont pas toutes la même efficacité.

\section{Les pièges alimentaires}

Ces pièges sont constitués d'une plaque de glu au milieu de laquelle est placé un appât alimentaire. Ces appâts sont à base de céréales, d'épices, de concentré de pruneau, de tourteau de

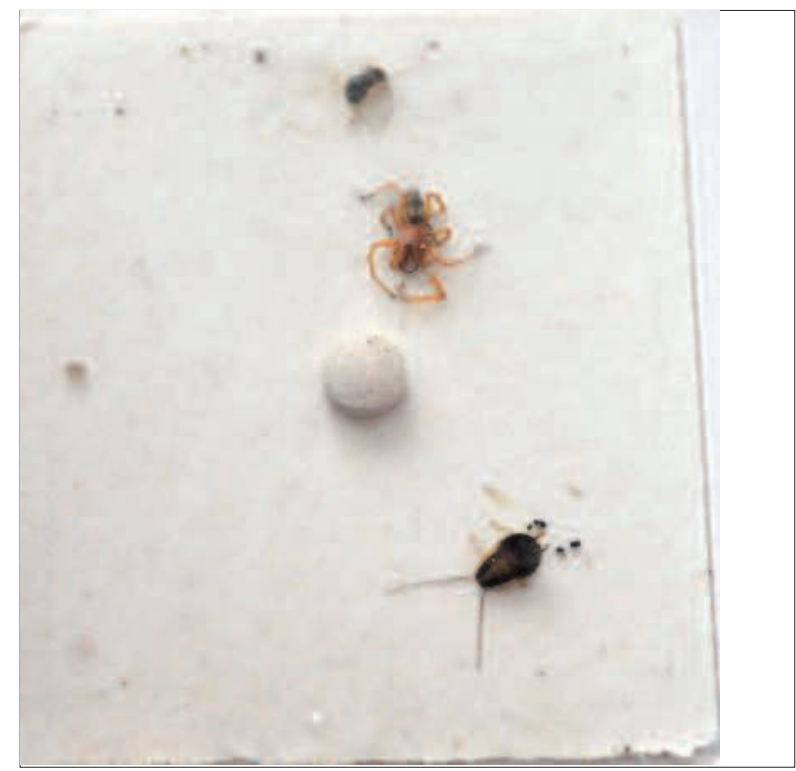

Piège alimentaire, constitué d'une pastille banche (au centre) à base d'épices, placée sur une plaque de glu et qui a permis la détection d'un poisson d'argent (en haut) et d'une blatte (en bas). 
noisette, ou de toutes autres substances fortement odorantes qui font partie du régime alimentaire de l'espèce d'insectes que l'on veut piéger. Ils sont à placer à proximité des œuvres dont on a repéré des dégradations ou non loin du lieu de visualisation des insectes (visualisation de la fuite rapide d'un poisson d'argent par exemple). Particulièrement adaptés pour le piégeage des insectes rampants, ils peuvent se placer discrètement au sol (plutôt le long des murs) sous un meuble, sous une étagère ou cachés dans une vitrine.

Ces pièges utilisés pour le piégeage des insectes volants et rampants sont particulièrement efficaces contre les blattes et les poissons d'argent.

\section{Les pièges à glu simple}

Ils sont constitués d'une simple plaque de glu pliée en forme de toit ou placée dans une boîte pour éviter un trop fort empoussièrement. Ils se positionnent sur le passage supposé des insectes et sont particulièrement adaptés pour le piégeage des insectes rampants. On les place en général le long des plinthes (les insectes se déplacent rarement au centre d'une pièce car ils se sentent en danger), sur le rebord d'une fenêtre ou sur une étagère. Ils peuvent être posés de part et d'autre d'une porte, ce qui permet de savoir si l'infestation provient de l'intérieur d'une salle ou de l'extérieur de celleci. Ils permettent de détecter la présence de blattes, de poissons d'argent et d'espèces de la sous famille des Ptininae (genre Ptinus, Niptus, Gibium...) ou tout autre insecte rampant présent dans l'environnement.

\section{Remarques générales \\ lors de l'implantation des pièges}

Il est important de noter que l'implantation de ces systèmes de piégeages dans un lieu, représente une alerte d'un

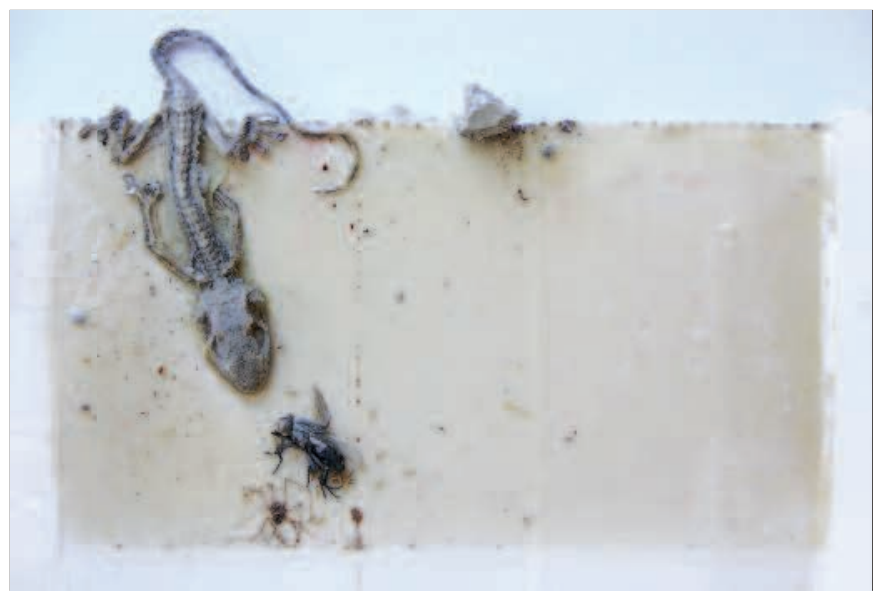

Piège à glu placé sur le passage des insectes ayant accidentellement piégé un lézard (une tarente), ce qui indique une ouverture importante sur l'extérieur. (c) CICRP problème d'infestation ou d'un défaut d'organisation interne au sein d'un établissement. Néanmoins, il n'est absolument pas opportun de placer des pièges « en préventif » si aucune dégradation ni aucun insecte n'ont été repérés au préalable car cela demande bien souvent des moyens humains et financiers non négligeables.

Pour être pleinement efficaces et permettre de donner l'ensemble des informations désirées, ces campagnes de piégeage devront faire l'objet d'un suivi particulier à savoir :

- référencer la localisation des pièges sur un plan et noter la date d'implantation et pour quel type d'insecte ceux-ci sont utilisés ;

- faire un planning pour le relevé des pièges et des insectes piégés (par exemple, tous les mois en période d'apparition des insectes, du printemps à l'automne, et tous les deux mois pour la période hivernale) ;

- faire un planning pour le changement des capsules attractives (pour les pièges à phéromone et les pièges alimentaires) car elles possèdent une durée d'action limitée dans le temps ;

- veiller à changer les plaques adhésives lorsqu'un grand nombre d'insectes se trouvent piégés (perte d'efficacité du piégeage).

En fonction de l'ampleur des infestations et afin de réaliser un suivi des populations d'insectes dans le temps (par rapport aux actions entreprises en aval), les campagnes de piégeage peuvent s'étaler sur plusieurs mois voire sur plusieurs années.

À partir des premiers résultats de piégeages obtenus, les aires d'implantation des pièges peuvent s'étendre (lorsque qu'il n'y a pas d'insecte piégé) à d'autres lieux annexes ou être réduites lorsque certains pièges sont fortement remplis d'insectes par rapport à des pièges «vides » sans insecte présents dans le même lieu.

Ces campagnes doivent faire partie d'un ensemble d'opérations conjointes (entretien des collections et des espaces, actions à mener sur les bâtiments, contrôle du climat...) afin de réduire les populations d'insectes dans un lieu. Elles doivent donc s'inscrire dans une démarche globale appelée IPM (Integrated Pest management) dans laquelle toutes les actions sont à mener conjointement et permettre in fine, la réduction significative des populations d'insectes dans un lieu (le terme « éradication » conviendrait davantage, mais dans ce domaine rien n'est jamais acquis).

\section{Comment identifier les insectes récoltés et à qui s'adresser?}

Actuellement la reconnaissance des insectes s'effectue par une identification morphologique des adultes qui requiert des clés d'identification complexes, différentes pour tous les groupes taxonomiques (coléoptères, diptères, lépidoptères...). Cette identification devient encore plus complexe 
lorsque l'on travaille sur les stades œuf, larve et nymphe du développement des insectes. Elle requiert alors très souvent la compétence de spécialistes tels que les entomologistes. Il est donc important de connaître des interlocuteurs qui ont une bonne connaissance des insectes dits «patrimoniaux » ou de pouvoir accéder à des collections entomologiques de référence. Ces collections sont souvent indispensables pour permettre la détermination d'un insecte que l'on compare avec ceux présents dans les boîtes de référence. Elles sont localisées au sein des muséums, des facultés des sciences, dans divers organismes (Institut national de la Recherche agronomique, Laboratoire national de la Protection des végétaux, Centre national de la Recherche scientifique, Institut de Recherche pour le Développement, Centre de coopération internationale en recherche agronomique pour le développement), ainsi que dans des associations et des sociétés entomologiques (Office pour les insectes et leur environnement) ou chez des entomologistes amateurs. Néanmoins ces collections de références sont parfois en mauvais état de conservation ou incomplètes, ce qui ne permet pas toujours d'obtenir une identification fiable.

Ces identifications peuvent aussi être réalisées par des restaurateurs-conseils formés en entomologie du patrimoine et par certaines entreprises privées qui travaillent dans le secteur du traitement et de la prévention. Ce travail d'identification peut être facilité par la consultation de bases de données en lignes qui regroupent les différents insectes présents dans les lieux patrimoniaux.

Dans l'idéal, ces identifications pourront être optimisées grâce aux actions suivantes :

- création d'une boîte d'insectes de référence par musée ou par ville qui regroupe les principaux insectes dangereux (environ
30 espèces) ce qui permettra de faire une comparaison visuelle avec les insectes prélevés ;

- réalisation d'une photographie (ou simple visualisation) de l'insecte prélevé à l'aide d'une loupe binoculaire (grossissement minimum de 40 fois) ou d'une mini caméra port USB ;

- identification de l'insecte prélevé par comparaison avec les insectes de référence en lien avec les clés d'identification présentes dans la base de données.

Ces bases de données sont consultables à partir des sites Internet de l'OCIM, de l'INRA ou du CICRP par exemple.

\section{Conclusion}

Les causes de la présence des insectes dans les lieux muséographiques sont multiples et variées et dépendent bien souvent de quelques facteurs, le niveau d'entretien des collections étant un facteur clé à leur implantation et leur pullulation. Ces causes ne peuvent être pleinement analysées que par l'identification des espèces d'insectes rencontrées dans ces lieux. Leur détection précoce suivie d'une identification fiable permet bien souvent d'entreprendre rapidement des actions qui limitent leur développement et leur propagation. Cette détection doit être menée par tous les acteurs d'une institution, du personnel d'entretien (qui remarque par exemple de la vermoulure fraîche) jusqu'aux personnes décisionnaires qui prennent rapidement des mesures ciblées (par exemple mise en quarantaine d'une œuvre nouvellement infestée). D’une bonne identification de ces insectes découleront les différentes actions spécifiques à mener pour permettre de maintenir les œuvres dans des conditions de conservation optimale.

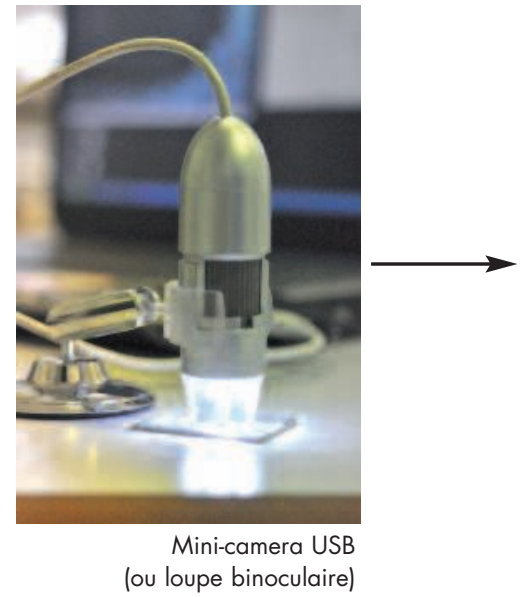

(ou loupe binoculaire)

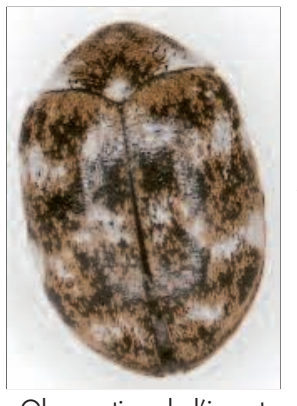

Observation de l'insecte prélevé (ou photo)
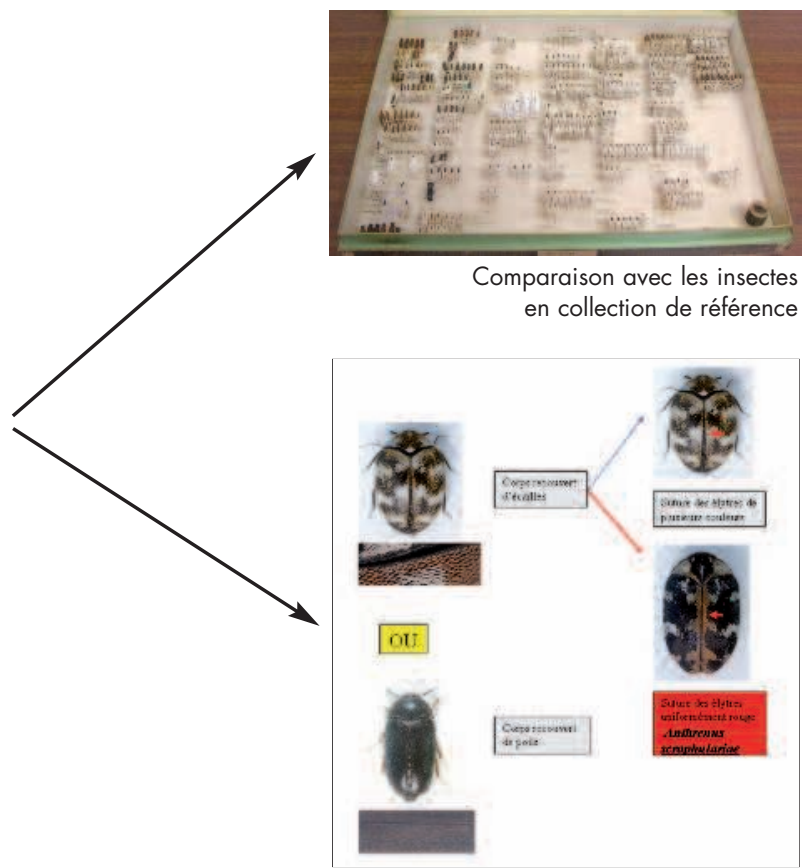

Identification de l'insecte à partir des clés de la base de données : ici début de la clé des Dermestidae 


\section{Bibliographie}

Cocquempot, C. et Valladarès, L. Datation des déprédations de termites et autres insectes xylophages de l'habitat et du bois d'œuvre, Le Cahier technique de l'INRA, vol. 67, 2009, pp. 31-42. Approche méthodologique intéressante pour mieux connaître la date du début d'une infestation d'insectes xylophages et notamment les vrillettes.

Colonna, J.-P. L'optimisation des pièges U.V. à insectes, Nuisibles et Parasites Information $\mathrm{n}^{\circ} 47$, octobre-novembre 2006, pp. 27-29.

D'Aguilar, J. et Fraval, A. Glossaire entomologique. Paris : Delachaux et Niestlé, 2004, 224 p.

Delobel, A. et Tran, M. Les coléoptères des denrées alimentaires entreposées dans les régions chaudes, Faune tropicale XXXII. Paris : Orstom/CTA, 1993, 425 p.

Delvare, G. et Aberlenc, H-P. Les insectes d'Afrique et d'Amérique tropicale : clés pour la reconnaissance des familles. CIRAD, Département GERDAT, Laboratoire de faunistique 1989, pp. 170-200.

Flieder, F., Capderou, C. et Duchein, M. Sawvegarde des collections du patrimoine : la lutte contre les détériorations biologiques. Paris : CNRS, 1999. 256 p.

Fohrer, F., Baslé, K. et Daniel, F. Problématique de l'infestation des colles de rentoilage des peintures de chevalet par le Stegobium paniceum (L.), Support tracé, n6, 2006, pp. 78-85.

Fohrer, F. et Martinez M. Première mention de Sefrania bleusei Pic, 1899 (Coleoptera, Dermestidae), pour la France et description de la femelle de l'espèce, Biocosme mésogéen, n²7 (3), 2010, pp. 102-107. Les auteurs y signalent pour la première fois, la présence en France du Coléoptère Demestidae Sefrania bleusei. Des éléments sont donnés sur sa morphologie, sa distribution géographique et sa biologie. La femelle, jusqu'alors non connue, est brièvement décrite. L'espèce pourrait représenter une menace pour les collections muséographiques (animaux naturalisés, collections d'insectes, collections ethnographiques).

Kingsley, H., Pinniger, D., Xavier-Rowe, A. et Winsor, P. Integrated pest management for collections : proceedings of 2001, a pest odyssey : 1-3 October 2001. Londres : James \& James, 2003. 150 p.

Laclos, E. (de) et Büche, B. La vrillette sans peine (Coleoptera Anobiidae), L'entomologiste, tome 64, $\mathrm{n}^{\circ} 1$, janvier-février 2008, pp. 3-10 ; tome $64, \mathrm{n}^{\circ} 4$, juillet-août 2008, pp. 217-220 ; tome 65, n 1 , janvier-février 2009, pp. 13 20 ; tome $65, n^{\circ} 4$, juillet-août 2009 , pp. 207-213. Pour tous ceux qui veulent en connaître un peu plus sur la famille des Anobiidae (vrillettes, insectes xylophages) avec un axe fort sur sa nouvelle classification.

Lepesme, P. Les coléoptères des denrées alimentaires et des produits industriels entreposés. Édition Lechevalier, 1944.

Martinez, M. et Dommanget, J.-L. Adresses utiles à l'entomologiste. Office pour les insectes et leur environnement, 2002, 96 p. (www.inra.fr).
Martinez, M. et Fohrer, F. Les insectes xylophages : qui sont-ils ? que mangent-ils ?, PHM-Revue horticole, n508, 2008, pp. 11-13.

Mary-Lou, F. Heritage Eaters, insects and fungi in heritage collections. Londres : James \& James, 1997.

Meunier, J.-J. Patrimoine culturel et altération biologique. Actes des journées d'études de la SFIIC-Poitiers, 17 et 18 novembre 1988, pp. 45-52. Sur quelques insectes déprédateurs des archives.

Zycherman, L.-A. et Schrock, J.-R. A guide to museum pest control. Washington, D.C. : Foundation of the American Institute for Conservation of Historic and Artistic Works, 1988, 205 p.

\section{Sites Internet}

\section{Fiches consultables sur le site du CICRP :}

- Le prélèvement des insectes : www.cicrp.fr/prelevement-insecte.html - De l'usage des pièges à lumière ultra violette pour surveiller les risques d'infestation- Fabien Fohrer-Fiche pratique du CICRP : www.cicrp.fr/pieges-insectes-uv.html

\section{Notes consultables sur le site de l'ICC :}

- www.cci-icc.gc.ca/publications/ccinotes/pdf-documents/n3-3_f.pdf - Stratégies de lutte préventive contre les infestations et méthodes de détection / Strang Thomas J.-K., note de l'ICC 3/1, 1996, 5 p.

- Détection des infestations : inspection des installations et liste de contrôle / Strang Thomas J.-K., note de l'ICC 3/2, 1996, 4 p.

Bases de données pouvant être utilisées pour la reconnaissance des insectes patrimoniaux :

- www.ocim.fr et www.cicrp.fr/insectes-du-patrimoine : sites facilitant la reconnaissance des insectes muséophages

- www.dermestidae.com : liste des Dermestidae européens avec galeries de photographies et détails anatomiques des différentes espèces

- www.insecte.org/forum/viewtopic.php?f=43\&t=2731\&view=next : liste des Dermestidae de France avec clés simplifiées

- www.inra.fr/opie-insectes/pa.htm : nombreuses informations et ouvrages sur les insectes

Base de données pour vérifier la validité des noms de genres et d'espèces d'insectes :

Fauna Europaea : www.faunaeur.org.

\section{Matériel :}

- aspirateur à insectes : www.eveiletjeux.com/.../aspirateur-a-insectes.htm ; www.scienceshopping.com/aspirateur-d-insectes-buki.fr

- boîtes à insectes : www.pyramide-entomologie.com ; www.cahurel-entomologie.com/boites_entomologiques.htm ; www.deyrolle.com

- épingles : www.skiclub.wz.cz/petr/p_fr.php ; www.kabatek.cz ; www.revol.fr/ index.asp?idgamme $=2$

- tout matériel : www.entomopraxis.com ; www.entomo.oxatis.com ; www. entosphinx.cz 